\title{
A single-institution experience with bevacizumab in the treatment of metastatic colorectal cancer and in conjunction with liver resection
}

This article was published in the following Dove Press journal:

OncoTargets and Therapy

I July 2014

Number of times this article has been viewed

\author{
Pia Osterlund ${ }^{1,2}$ \\ Reetta Peltonen ${ }^{2,3}$ \\ Tuomo Alanko' \\ Petri Bono ${ }^{1,2}$ \\ Helena Isoniemi ${ }^{2,3}$ \\ 'Department of Oncology, Helsinki \\ University Central Hospital, Helsinki, \\ ${ }^{2}$ Institute of Clinical Medicine, \\ University of Helsinki, Helsinki, \\ ${ }^{3}$ Department of Surgery, Helsinki \\ University Central Hospital, Helsinki, \\ Finland
}

Background: Bevacizumab is active in the treatment of metastatic colorectal cancer (mCRC). However, efficacy of bevacizumab has predominantly been evaluated on selected patients with relatively good performance status and minor comorbidities. We evaluated the efficacy and safety of bevacizumab in unselected patients with $\mathrm{mCRC}$, some of whom underwent liver resection.

Material and methods: All patients with inoperable mCRC, fit for combination chemotherapy $(n=180)$, who were initially not resectable, not included into studies and without contraindications to bevacizumab, and initiated on bevacizumab at the Helsinki University Central Hospital between April 2004 and December 2005 were included $(n=114)$. Most $(n=70)$ received 5-fluorouracil/ leucovorin/irinotecan plus bevacizumab as first-line therapy. The remainder $(n=44)$ of the patients received bevacizumab in combination with oxaliplatin or irinotecan with or without 5 -fluorouracil or capecitabine. Minimum follow-up was 7 years. Treatment response was evaluated every $8-10$ weeks according to RECIST criteria.

Results: Median age was 59.6 years (range 35-79); male/female ratio was 54\%/46\%; World Health Organization performance status $0 / 1 / 2-3$ was $33 \% / 55 \% / 11 \%$, respectively; and the number of metastatic sites, one/two/three or more, was $31 \% / 21 \% / 48 \%$, respectively. Median duration of bevacizumab therapy was 7.8 months (range $0.5-70.5$ with pauses). In first-line $(n=40)$, response rate (RR) was 62\%, progression-free survival (PFS) 11.7 months, and overall survival (OS) 22.1 months. In second-line ( $\mathrm{n}=43$ ), RR was 44\%, PFS 8.7 months, and OS 18.7 months. In later lines $(n=31)$, RR was 14\%, PFS 6.7 months, and OS 14.2 months. Ten patients with initially unresectable liver metastases became operable and R0 resection was achieved in $90 \%$ (9/10 resections). In 23\% (7/31) of operated metastases, no vital tumor cells were found in histologic examination. Operative morbidity was low: two mild infections, no increased bleeding tendency was noticed, and no impaired wound healing occurred.

Discussion: Bevacizumab-containing combination therapy was effective with acceptable tolerability in an unselected $\mathrm{mCRC}$ patient population in which liver resections could be safely performed.

Keywords: everyday clinical practice, colorectal cancer, metastatic, liver resection

\section{Introduction}

Colorectal cancer (CRC) remains an urgent health concern, being the second most common cancer worldwide, despite improvements in systemic chemotherapy. For example, in Finland in 2010 there were 2,744 new cases of CRC. Bevacizumab (Avastin $^{\circledR}$; Genentech/Roche, Basel, Switzerland) is a recombinant humanized monoclonal antibody with selectivity against vascular endothelial growth factor that is approved for use as first- and second-line treatment of metastatic CRC (mCRC). Overall survival benefit in mCRC was found with bevacizumab in three Phase III
Correspondence: Pia Osterlund Department of Oncology, Helsinki University Central Hospital, PO Box I80, 00029 HUS (Helsinki), Finland

Tel +358947 II

Fax +358947174202

Email pia.osterlund@hus.fi 
studies and one combination analysis of three studies. ${ }^{1-4}$ Benefits with bevacizumab in $\mathrm{mCRC}$ have been reported when used in combination with irinotecan/5-fluorouracil (5-FU)/leucovorin (LV) (IFL), ${ }^{1}$ 5-FU/LV, ${ }^{3}$ capecitabine/oxaliplatin (XELOX), and 5-FU/LV/oxaliplatin (FOLFOX). ${ }^{2,5}$ These findings were supported by survival data from large patient registry and postmarketing surveillance studies carried out in the US and Europe (BRiTE, FirstBEAT and ARIES). ${ }^{6-8}$

A number of patient- and disease-related factors may need to be considered when evaluating candidates for bevacizumab-related therapy, such as history of recent severe arterial thromboembolic events ${ }^{9,10}$ or venous thrombosis, ${ }^{1,10}$ history of gastrointestinal (GI) perforation, ${ }^{3,7,11}$ noncontrolled hypertension, ${ }^{1,3,12}$ and resectability of liver metastases. ${ }^{1,5,7,13-15}$ Data regarding liver resections of cetuximab- and bevacizumab-treated patients were limited in 2004-2005 ${ }^{16}$ and, therefore, borderline resectable patients received chemotherapy with FOLFOX. Much of the randomized data on bevacizumab were on relatively young first-line patients with excellent performance status and usually only one metastatic site, which is unlike the clinical situation facing many oncologists.

The aim of this retrospective single-institution analysis was to evaluate the safety and efficacy of bevacizumab treatment and feasibility of liver resection in a cohort of unselected $\mathrm{mCRC}$ patients initiating bevacizumab-containing chemotherapy between April 2004 and December 2005.

\section{Patients and methods Retrospective analysis of the use of bevacizumab in $\mathrm{mCRC}$}

The findings from a retrospective analysis of the use of bevacizumab at the Department of Oncology, Helsinki University Central Hospital ( $\mathrm{HUCH}$ ) (catchment area 1.5 million inhabitants) are reported.

Bevacizumab has been used as a standard treatment in mCRC at $\mathrm{HUCH}$ since April 2004. During the inclusion period (April 2004 to December 2005), 825 cases of CRC were diagnosed (Figure 1). Of these 825 patients, 225 were fit for chemotherapy for $\mathrm{mCRC}$, of whom 45 were given single agents (due to age and poor performance status or patient preference) leaving 180 that started combination chemotherapy and were considered for bevacizumab treatment. Further exclusion was due to neoadjuvant chemotherapy for potentially resectable disease $(n=20)$, inclusion into trials $(n=16)$, and contraindications for bevacizumab treatment $(n=30$; brain metastases, high dose nonsteroidal anti-inflammatory drugs, prior pulmonary embolism, recent venous thromboembolic event, and prior arterial thromboembolic event or baseline proteinuria).

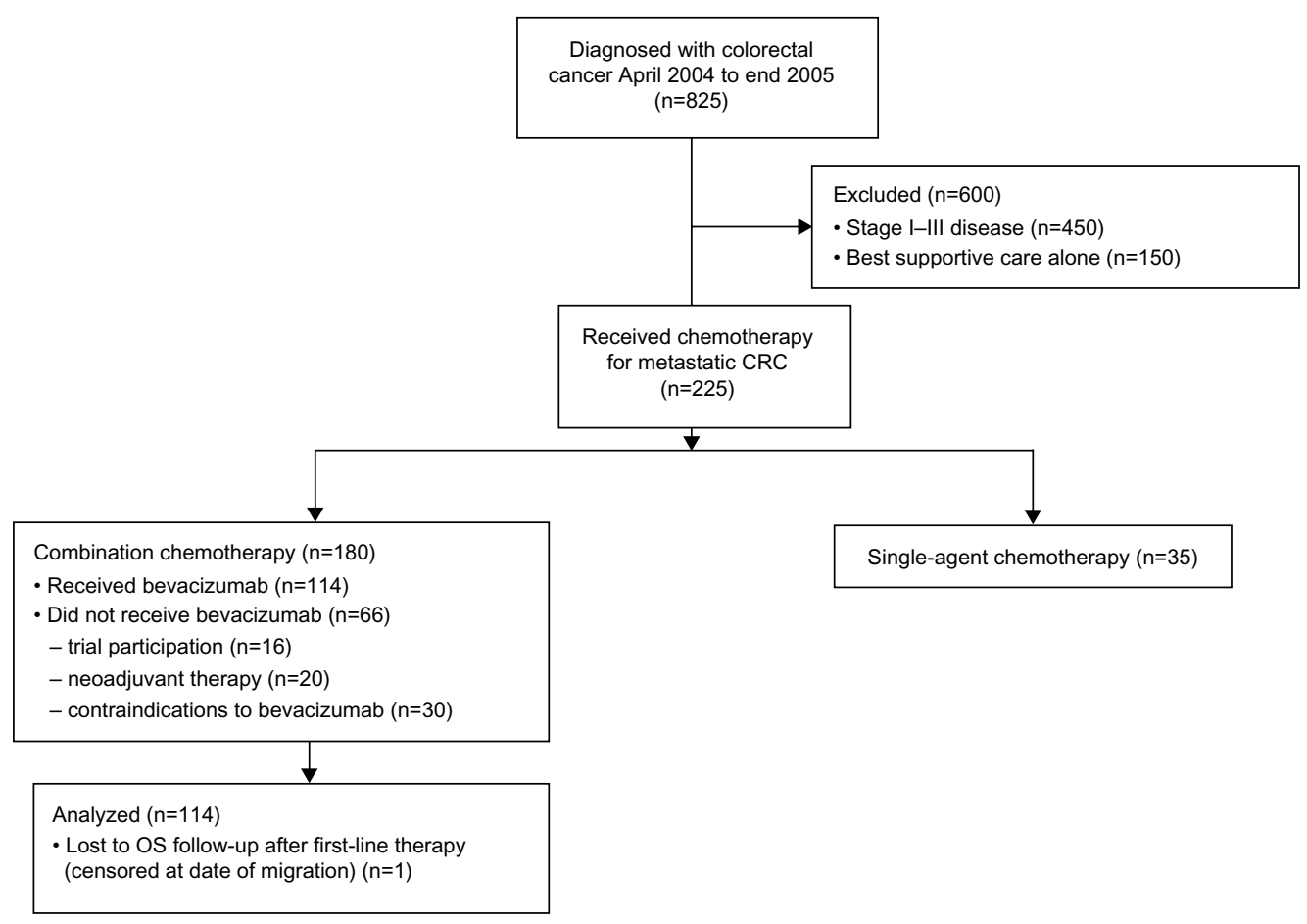

Figure I Treatment algorithm for colorectal cancer cases during study period (April, 2004-December, 2005) at the Helsinki University Central Hospital district (I,424,965 inhabitants in January 2004).

Abbreviations: CRC, colorectal cancer; OS, overall survival. 
All patients diagnosed with inoperable $\mathrm{mCRC}$ and initiating treatment with bevacizumab at $\mathrm{HUCH}$ between April 2004 and December 2005 were included in the analysis $(\mathrm{n}=114)$. During that time, bevacizumab-containing therapy was not routinely used in patients considered for liver or lung resection. However, ten of these 114 (9\%) initially inoperable patients subsequently underwent resection of liver metastases after having been treated with bevacizumab-containing chemotherapy.

The standard first-line therapy for mCRC at $\mathrm{HUCH}$ was 5-FU/LV/irinotecan (FOLFIRI) plus bevacizumab; most patients $(\mathrm{n}=70)$ received FOLFIRI plus bevacizumab as first-line therapy. In those patients who had not previously received bevacizumab, and when FOLFIRI was considered contraindicated as second- or later-line therapy, bevacizumab was added to irinotecan-, oxaliplatin-, or 5-FUbased therapy, depending on the previous chemotherapy regimen. Irinotecan-based therapy was used in 22 patients (capecitabine/irinotecan, IFL, or single irinotecan), oxaliplatin-based therapy in 12 patients (FOLFOX or XELOX), and 5-FU-based therapy in nine patients (5-FU/ LV modified de Gramont regimen or capecitabine). One dihydropyrimidine dehydrogenase-deficient patient received single-agent bevacizumab.

Data from all patients starting bevacizumab therapy between April 2004 and December 2005 were collected. Data cut-off was set at September 4, 2013 for safety and efficacy, which resulted in a minimum follow-up of 7.7 years for the six patients alive; one patient was lost to follow-up. Patient demographics, adverse events, hospitalizations, and treatment efficacy were recorded and collated. All cancer treatments before, during, and after bevacizumab were recorded, including chemotherapy, radiotherapy, and operations. Treatment response was evaluated according to RECIST criteria by whole body spiral computerized tomography (CT) performed every $8-10$ weeks. The data monitoring system and procedure was approved by $\mathrm{HUCH}$, and the vast majority of patients were treated by Doctors Osterlund and Alanko.

Patients considered for liver resection were thoroughly evaluated prior to resection with liver ultrasound, whole body $\mathrm{CT}$, magnetic resonance imaging, or positron-emission tomography scan (when clinically indicated) and laboratory tests. Laboratory tests included liver enzymes, bilirubin, albumin, prealbumin, cholesterol, thromboplastin time, galactose eliminating test, and tumor markers. Patients were evaluated by a multidisciplinary team before resection, perioperatively, and at 3 months postoperatively.

\section{Statistical analyses}

All statistical analyses were performed using a StatView statistical package (Berkley, CA, USA) and sigma-plot version 11.0 (SysStat Software GmbH, San Jose, CA, USA). Nonnormal distributions were compared with the MannWhitney $U$-test. Survival analyses were carried out using the Kaplan-Meier method. Frequency tables were analyzed using the $\chi^{2}$ test. Progression-free survival (PFS) was defined as the time from the date of initiation of the bevacizumabcontaining therapy to the day of documented disease progression or death due to any cause, or was censored at last follow-up. Overall survival (OS) was defined as the time from the date of initiation of bevacizumab-containing therapy to the date of death or was censored at last follow-up. The data were stratified by the line of treatment.

\section{Results}

\section{Patient-population evaluated}

In total, 114 patients who initiated bevacizumab-containing treatment for mCRC between April 2004 and December 2005 at $\mathrm{HUCH}$, Helsinki, Finland were evaluated. Patient baseline demographics are shown in Table 1. Overall, the median age at bevacizumab initiation was 59.6 years (range $35-79$ ); $54 \%$ of patients were males and $46 \%$ females. The primary tumor site was colon in $56 \%$ and rectum in $44 \%$ of patients. The baseline

Table I Patient baseline demographics by line of treatment

\begin{tabular}{|c|c|c|c|c|}
\hline & $\begin{array}{l}\text { All } \\
(n=|| 4)\end{array}$ & $\begin{array}{l}\text { Ist line } \\
(n=40)\end{array}$ & $\begin{array}{l}\text { 2nd line } \\
(n=43)\end{array}$ & $\begin{array}{l}\text { 3rd to } 7 \text { th } \\
\text { line }(n=3 I)\end{array}$ \\
\hline Median age, & 59.6 & 62.6 & 57.2 & 59.5 \\
\hline years (range) & $(35-79)$ & $(35-78)$ & $(35-79)$ & $(39-76)$ \\
\hline \multicolumn{5}{|l|}{ Sex, \% } \\
\hline Male/female & $54 / 46$ & $43 / 57$ & $53 / 47$ & $68 / 32$ \\
\hline \multicolumn{5}{|l|}{ Primary site, \% } \\
\hline Colon & 56 & 65 & 58 & 42 \\
\hline Rectum & 44 & 35 & 42 & 58 \\
\hline \multicolumn{5}{|l|}{ WHO PS, \% } \\
\hline 0 & 33 & 35 & 35 & 29 \\
\hline I & 55 & 55 & 53 & 55 \\
\hline $2-3$ & II & 8 & 12 & 16 \\
\hline \multirow{2}{*}{\multicolumn{5}{|c|}{$\begin{array}{l}\text { Number of } \\
\text { metastatic sites, \% }\end{array}$}} \\
\hline & & & & \\
\hline I & 31 & 32 & 30 & 29 \\
\hline 2 & 21 & 20 & 26 & 16 \\
\hline $3+$ & 48 & 48 & 44 & 55 \\
\hline \multicolumn{5}{|l|}{ Liver metastases } \\
\hline Present & 72 & 70 & 74 & 71 \\
\hline Liver only site & 20 & 20 & 23 & 19 \\
\hline \multirow{2}{*}{\multicolumn{5}{|c|}{$\begin{array}{l}\text { Resection of } \\
\text { primary site, } \%\end{array}$}} \\
\hline & & & & \\
\hline No/yes & $83 / 17$ & $85 / 15$ & $84 / 16$ & $77 / 23$ \\
\hline
\end{tabular}


World Health Organization (WHO) performance status (PS) was 0 in 33\%, PS 1 in 55\%, and PS $2-3$ in 11\% of patients. Almost half of the patients $(48 \%)$ had three or more metastatic sites, and an unresected primary tumor or local recurrence was present in $17 \%$ of patients. Details of patients' previous therapy (radiotherapy, adjuvant therapy, chemotherapy) stratified by the line of treatment are shown in Table 2.

\section{Treatment duration}

The median duration of bevacizumab therapy in the entire cohort of 114 patients was 7.8 months (range $0.5-70.5$ with pauses). The median duration of therapy for the 70 patients who received bevacizumab plus FOLFIRI was 8.1 months (range $0-32$ ), and for the 44 patients who received bevacizumab plus other therapy it was 5.5 months (range 0-29). Analysis by line of treatment revealed that the patients who received bevacizumab as first-line treatment $(n=40)$ had a median duration of therapy of 8.3 months (range $0-15$ ) compared with 5.7 (range 0-21) and 6.4 months (range 0-22) for those who received it as second- $(n=43)$ and third-toseventh-line $(\mathrm{n}=31)$, respectively.

Table 2 Patients' previous therapy (radiotherapy, adjuvant therapy, chemotherapy) at entry by line of treatment and therapy in further lines of therapy after bevacizumab failure

\begin{tabular}{|c|c|c|c|c|}
\hline & $\begin{array}{l}\text { All } \\
(n=\mid 14)\end{array}$ & $\begin{array}{l}\text { Ist line } \\
(n=40)\end{array}$ & $\begin{array}{l}\text { 2nd line } \\
(n=43)\end{array}$ & $\begin{array}{l}\text { 3rd to } 7 \text { th } \\
\text { line }(n=3 I)\end{array}$ \\
\hline \multicolumn{5}{|l|}{ Radiotherapy, \% } \\
\hline No & 69 & 80 & 68 & 58 \\
\hline $5 \times 5$ Gy & 16 & 15 & 16 & 16 \\
\hline 45-50 Gy & 15 & 5 & 16 & 26 \\
\hline \multicolumn{5}{|c|}{ Adjuvant therapy, \% } \\
\hline No & 71 & 75 & 70 & 67 \\
\hline 5-FU-based & 26 & 25 & 21 & 32 \\
\hline Oxaliplatin & 4 & 0 & 9 & 0 \\
\hline \multicolumn{5}{|l|}{ Pre-bevacizumab } \\
\hline \multicolumn{5}{|l|}{ chemotherapy, \% } \\
\hline No & 35 & 100 & 0 & 0 \\
\hline 5-FU-based & 65 & 0 & 100 & 100 \\
\hline Irinotecan & 35 & 0 & 28 & 90 \\
\hline Oxaliplatin & 46 & 0 & 58 & 87 \\
\hline Other* & 8 & 0 & 5 & 29 \\
\hline \multicolumn{5}{|c|}{ Post-bevacizumab } \\
\hline \multicolumn{5}{|c|}{ chemotherapy, \% } \\
\hline No & 30 & 25 & 26 & 42 \\
\hline 5-FU-based & 48 & 50 & 56 & 32 \\
\hline Irinotecan & 20 & 15 & 26 & 19 \\
\hline Oxaliplatin & 25 & 38 & 21 & 13 \\
\hline Cetuximab & 18 & 13 & 21 & 23 \\
\hline Bevacizumab & 17 & 25 & 14 & 10 \\
\hline Other* & 4 & 5 & 2 & 6 \\
\hline
\end{tabular}

Note: *Other chemotherapy included raltitrexed, gemcitabine, mitomycin C, taxanes, PTK787, and erlotinib.

Abbreviations: 5-FU, 5-fluorouracil; Gy, Gray.

\section{Clinical efficacy of bevacizumab in $\mathrm{mCRC}$}

In all 114 patients, the response rate (RR) was $42 \%$ and the disease control rate was $89 \%$. The median PFS was 8.8 months and the median OS was 18.4 months.

In first-line patients ( $\mathrm{n}=40)$, RR was $62 \%$ and the median PFS 11.7 months and three patients (8\%) were in remission at the minimum of 7.7 years (Figure 2). Median OS time for the first-line patients was 22.1 months, and $13 \%$ of patients were still alive at the minimum of 7.7 years (data cut-off, September 4, 2013) (Figure 3). In secondline patients ( $n=43), R R$ was $44 \%$ and the median PFS was 8.2 months (Figure 2). The median OS in second-line therapy was 18.7 months and one patient (2\%) was alive at data cut-off (Figure 3). In third- to seventh-line patients ( $\mathrm{n}=31$ ), RR was $14 \%$, PFS was 6.7 months (Figure 2), and OS was 14.2 months (Figure 3).

Evaluation of the best response to treatment in the overall population and by line of treatment revealed that the RR was higher among patients who received bevacizumab as part of first-line therapy versus second- or later-line therapies ( $P=0.012$ for first- versus second- versus third-line therapy; Table 3). For both PFS and OS, there was a significant trend of bevacizumab being more effective when given early (ie, as first-line rather than as second- or later-line treatment; PFS $P=0.037$ and $\mathrm{OS} P<0.001$ ).

Further chemotherapy after progression on bevacizumab was given to $70 \%$ of patients (Table 2). All of the three most important chemotherapeutic agents for colorectal

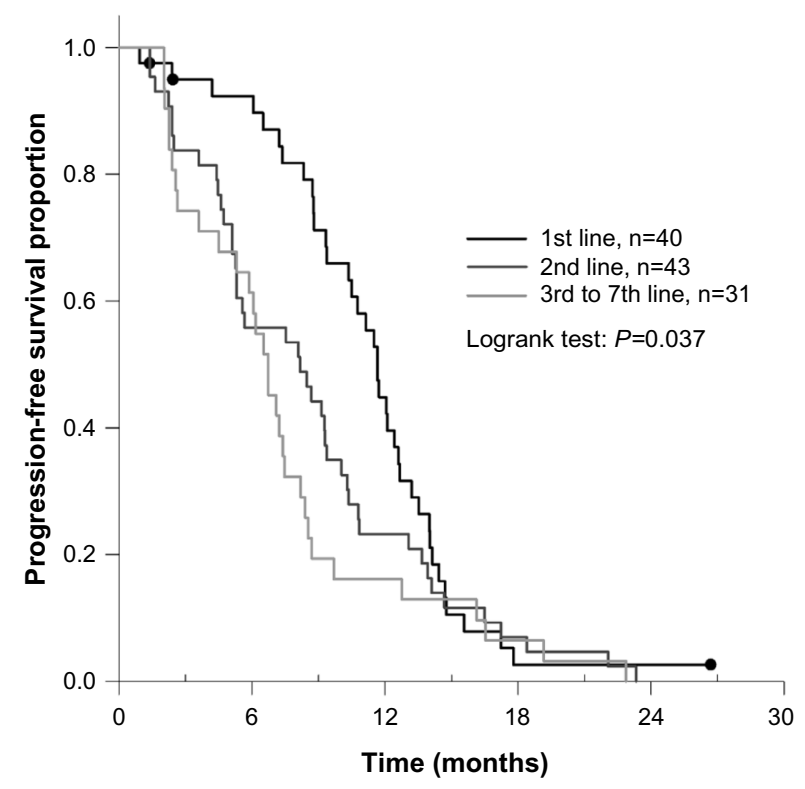

Figure 2 Progression-free survival with chemotherapy involving bevacizumab in $C R C$ patients by line of treatment.

Abbreviation: CRC, colorectal cancer. 


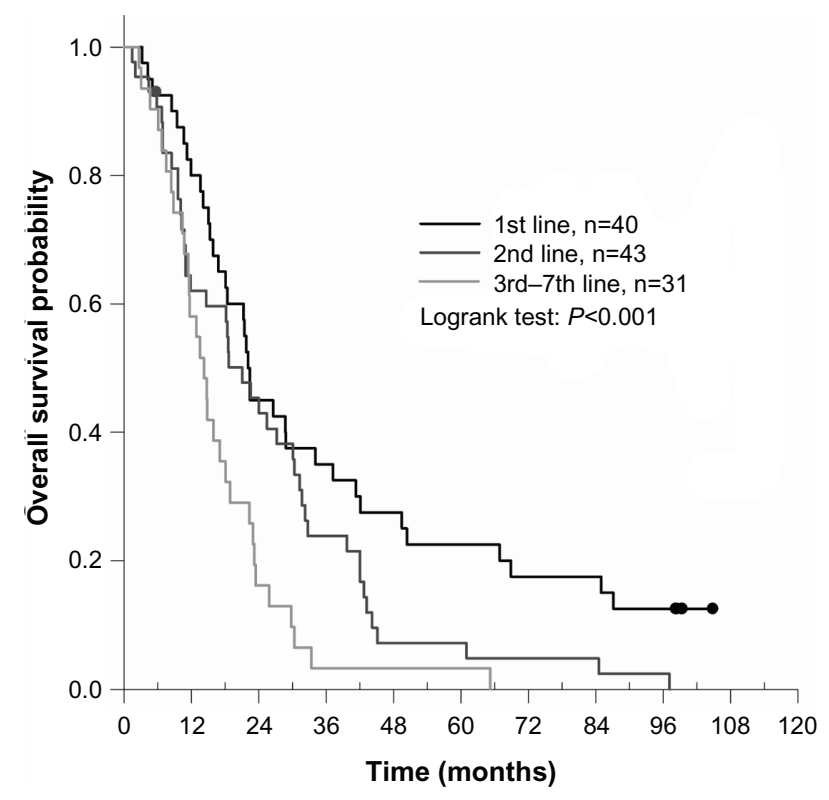

Figure 3 Overall survival with chemotherapy involving bevacizumab in $\mathrm{mCRC}$ patients by line of treatment.

Abbreviation: $\mathrm{mCRC}$, metastatic colorectal cancer.

adenocarcinoma (5-FU, oxaliplatin, and irinotecan) were used at some phase in the treatment in $69 \%$ of the patients. Cetuximab was given to $18 \%$ of the patients and their median OS was 39.7 months. Bevacizumab beyond progression was given to $17 \%$ of patients with a median OS of 32.7 months. Seven patients $(6 \%)$ responded to first line "stop-and-go" irinotecan, 5-FU, and bevacizumab therapy for more than 5 years (median 6.4 years, range 5.5-8.0).

\section{Clinical safety of bevacizumab in mCRC}

The safety profile with bevacizumab was as anticipated with adverse events and their severity being as predicted. There were no treatment-related deaths. The majority of bevacizumab-related adverse events were bleeding/ ulceration, arterial thromboembolic events, and perforation, with most of these being no more than grade 2 in severity (Table 4). The majority of adverse events reported during

Table 3 Patients' treatment response by line of treatment (I05 patients evaluable for response)

\begin{tabular}{lllll}
\hline & $\begin{array}{l}\text { All } \\
(\mathbf{n = I | 4 )}\end{array}$ & $\begin{array}{l}\text { Ist line } \\
(\mathbf{n = 4 0})\end{array}$ & $\begin{array}{l}\text { 2nd line } \\
(\mathbf{n}=\mathbf{4 3})\end{array}$ & $\begin{array}{l}\text { 3rd to 7th } \\
\text { line }(\mathbf{n = 3})\end{array}$ \\
\hline Complete response & $4 \%$ & $5 \%$ & $5 \%$ & $0 \%$ \\
Partial response & $38 \%$ & $57 \%$ & $39 \%$ & $14 \%$ \\
Stable disease & $47 \%$ & $33 \%$ & $49 \%$ & $62 \%$ \\
Progressive disease & $11 \%$ & $5 \%$ & $7 \%$ & $24 \%$ \\
Median PFS, months & 8.8 & 11.7 & 8.7 & 6.7 \\
Median OS, months & 18.4 & 22.1 & 18.7 & 14.2 \\
\hline
\end{tabular}

Abbreviations: PFS, progression-free survival; OS, overall survival.
Table 4 Incidence of bevacizumab-related adverse events and laboratory abnormalities in patients treated for $\mathrm{mCRC}(\mathrm{n}=\mathrm{I}$ |4)

\begin{tabular}{lll}
\hline Adverse event, \% & Grade I/2 & Grade 3/4 \\
\hline Hypertension & 12 & $\mathrm{I}$ \\
Proteinuria & 28 & $\mathrm{I}$ \\
Bleeding & 23 & 4 \\
Arterial thromboembolic events & 2 & $\mathrm{I}$ \\
Gl perforation & 0 & 3 \\
Diarrhea & 26 & 8 \\
Stomatitis & 26 & 3 \\
Nausea & 47 & 4 \\
Fatigue & 53 & $\mathrm{I}$ \\
Hand-foot syndrome & 19 & $\mathrm{I}$ \\
Neuropathy & 17 & 3 \\
Alopecia & 27 & $\mathrm{~N} / \mathrm{A}$ \\
Anemia & 62 & 0 \\
Neutropenia & 30 & 32 \\
Thrombocytopenia & 34 & 2 \\
\hline
\end{tabular}

Abbreviations: $\mathrm{Gl}$, gastrointestinal; $\mathrm{mCRC}$, metastatic colorectal cancer; N/A, not applicable.

combination chemotherapy were likely due to the cytotoxic chemotherapy partner.

Serious adverse events were as predicted with no difference between lines of treatment in the proportion of patients requiring hospitalization for any reason (all patients: $39 \%$ had in-hospitalizations; first-line: 18 patients [45\%]; second-line: 15 patients [35\%]; third-line: 12 patients [39\%]). Chemotherapy-related toxicity led to hospitalizations in 41 patients $(36 \%)$ and bevacizumab-related events (GI perforation, arterial thromboembolic event, or rectal bleed) in eleven patients (10\%). The remainder (seven patients, $6 \%$ ) were hospitalized due to constitutional symptoms caused by disease progression or due to elective surgery.

\section{Use of bevacizumab in initially unresectable liver metastases prior to liver resection}

In 2004, no data regarding liver resection of bevacizumabtreated patients were available, and potentially resectable patients were given neoadjuvant FOLFOX chemotherapy. Only patients with nonresectable disease and high tumor burden initiated bevacizumab-containing therapy. Only 20\% of patients had liver-only disease (Table 1). Ten out of 114 patients $(9 \%)$ who had started chemotherapy combined with bevacizumab in 2004-2005 with initially nonresectable liver metastases subsequently underwent liver resection.

These 10 patients had a total of 66 metastases, visualized by radiological examinations prior to initiation of bevacizumab and chemotherapy. Upon CT-scan within 4 weeks prior to resection, only 35 (53\%) of these lesions could be found. In the operating theatre, 32 of the 66 (48\%) 
metastases were identified by palpation and peroperative ultrasound. Of these, 31 were resected and one was destroyed with radiofrequency ablation. The median number of removed metastases per patient was three (range one to six) and the median size of the biggest lesion was $1.9 \mathrm{~cm}$ (range 0.4-6.0). R0 resections were achieved in 90\% (nine out of ten) of operations, and 23\% (seven out of 31 ) of lesions did not contain vital tumor cells in rigorous histological examination.

Bevacizumab was interrupted for a median of 47 days (range 36-196) before surgery and restarted a median of 30 days (range 27-60) after resection. Median inhospitalization time was 8 days (range 7-14). Morbidity was low: bleeding was limited with median perioperative erythrocyte transfusion of 1 unit (range 0-6); two mild infections were noted; and wound healing was not impaired. Two patients experienced reversible atrial fibrillation postoperatively. Three months after resection, liver regeneration had occurred as anticipated; liver synthesizing capacity, liver enzymes, and CT control were back to baseline (Table 5).

Re-resections have been performed in three patients. Altogether, 13 liver resections have been performed in these patients. The re-resections were performed 9-14 months after the previous resection. All four re-resections were performed safely and the postoperative course has been as anticipated. One of the ten patients who underwent liver resection is presented in Figure 4.

Table 5 Key laboratory findings in ten patients at first liver resections preoperatively, at discharge after liver resection, and at 3 months postoperatively

\begin{tabular}{|c|c|c|c|}
\hline $\begin{array}{l}\text { Liver tests } \\
\text { (reference } \\
\text { range) }\end{array}$ & Preoperative & At discharge & $\begin{array}{l}3 \text { months } \\
\text { postoperative }\end{array}$ \\
\hline ALT, U/L & $13(10-19)$ & $63(5 I-132)$ & $22(18-23)$ \\
\hline AFOS, U/L & $74(52-155)$ & $145(76-173)$ & II 8 (75-I88) \\
\hline $\begin{array}{l}\text { Total bilirubin, } \\
\mu \mathrm{mol} / \mathrm{L}\end{array}$ & $10(9-12)$ & $10(9-10)$ & II (9-13) \\
\hline TT-SPA, \% & $110(98-130)$ & $57(5 I-73)$ & $106(98-110)$ \\
\hline $\begin{array}{l}\text { Galactose } \\
\text { elimination, } \\
\text { TI/2 (minutes) }\end{array}$ & $12.8(\mid 1.7-16.6)$ & $19.5(15.9-23.5)$ & Not done \\
\hline Albumin, mg/L & $40.3(37-4 I)$ & $34.8(31-36)$ & $36.6(35-40)$ \\
\hline Prealbumin, mg/L & $261(222-276)$ & $120(100-142)$ & $243(209-28 I)$ \\
\hline $\begin{array}{l}\text { Cholesterol, } \\
\mathrm{mmol} / \mathrm{L}\end{array}$ & $5.0(4.4-6.0)$ & $3.1(2.8-3.3)$ & $4.9(4.8-5.2)$ \\
\hline CEA, kU/L & $3.5(1.7-4.7)$ & $1.8(1.2-3.6)$ & $2.5(1.2-5.7)$ \\
\hline $\mathrm{Ca} 19-9, \mathrm{kU} / \mathrm{L}$ & $7(I-12)$ & $12(9-20)$ & $6(4-9)$ \\
\hline
\end{tabular}

Note: Data are presented as median with interquartile range.

Abbreviations: ALT, alanine aminotransferase; AFOS, alkaline phosphatase; Ca 19-9, Cancer Antigen 19-9; CEA, carcinoembryonic antigen; TT-SPA, thromboplastin time; $\mathrm{TI} / 2$, half-life.

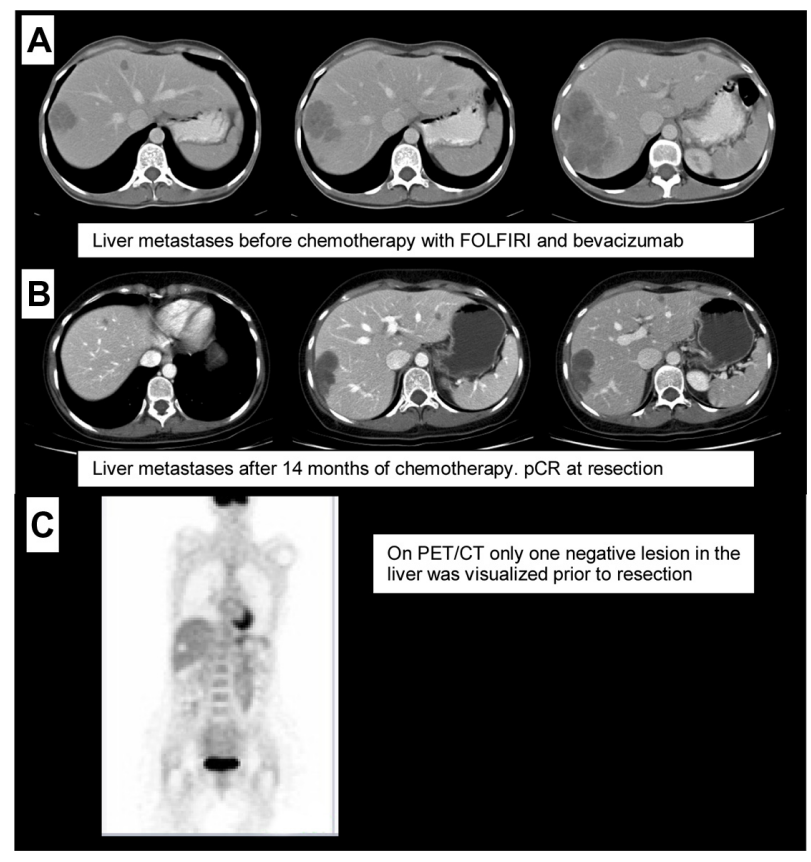

Figure 4 A 42 year old female patient with lung metastases and four synchronous liver metastases.

Notes: A rectal cT4N2MI disease was diagnosed in October 2004 (A). After 14 months of FOLFIRI plus bevacizumab chemotherapy and chemoradiation, radiologic complete response was noted in lung metastases and the patient underwent anterior resection in August 2006 with a pathologic complete response in histology. Liver resection was performed in October 2006 and CT findings preoperatively showed four metastases (B). In histology, no vital tumor cells were found in the resected liver tissue, including all four metastases. In 7 year follow-up, no signs of recurrence in liver or lung have been noted. One FDG-negative lesion was seen prior to resection on PET/CT scan (C).

Abbreviations: CT, computerized tomography; FOLFIRI, 5-fluorouracil/ leucovorin/irinotecan; $\mathrm{PCR}$, pathologic complete response; $\mathrm{PET}$, positron emission tomography; FDG, fluoro-deoxyglucose.

Median survival for the ten out of 114 resected patients was 23.2 months (interquartile range 18-69 months) and 18.4 months for the 94 non-resected patients (interquartile range 11-31, $P=0.09$ ).

\section{Discussion}

This single-institution analysis demonstrates that the safety and efficacy of bevacizumab in the everyday clinical practice setting (ie, a cohort of unselected mCRC patients) is in line with randomized trials. Focusing on the safety profile of bevacizumab, there were no treatment-related deaths, and bevacizumab-related adverse events were as anticipated (bleeding/ulceration, arterial thromboembolic events, and GI perforation), with most being not more than grade 2 in severity. These adverse effects were manageable in everyday practice.

The PFS and OS found in this study in patients who received bevacizumab as part of first-line therapy (11.7 and 22.1 months, respectively) is in line with data reported from the BRiTE registry (PFS 10.1 months; OS 27.1 months) ${ }^{6}$ and 
the FirstBEAT trial (PFS 10.8 months; OS 22.7 months). ${ }^{7}$ However, in contrast to these two studies, the patients reported on here had a higher tumor burden ( $48 \%$ of patients with three or more metastatic sites) and 55\% had WHO PS of 1 (and 11\% WHO PS 2-3), compared with $42 \%{ }^{6}$ and $34 \%{ }^{7}$ of patients with a PS of 1 in the other two trials. Interestingly, the safety profiles of bevacizumab reported here and in the FirstBEAT study were similar, with the major grade 3/4 bevacizumab-related adverse events of bleeding/ulceration, GI perforation, and arterial thromboembolic events all reported with a difference in frequency of less than $1.5 \%$ between the studies. ${ }^{7}$

The clinical efficacy of bevacizumab-containing regimens in first-line mCRC varied relatively little with the chemotherapy backbone used. These findings are supported by randomized clinical trials where bevacizumab was used in combination with 5-FU/LV, ${ }^{3,5}$ IFL, ${ }^{1}$ FOLFIRI, ${ }^{7,12}$ FOLFOX, ${ }^{2,5,6,12}$ and XELOX..$^{5-7,17}$ Again, the suggested OS and PFS advantages of bevacizumab in combination with chemotherapy in this retrospective analysis of 22.1 and 11.7 months, respectively, are in close agreement with the published literature with IFL, FOLFOX, and FOLFIRI. ${ }^{1-7,18}$ This indicates that the efficacy of bevacizumab in a clinical trial population translates into efficacy in the everyday clinical practice setting in an unselected patient population, which is reassuring.

As a result of this being an unselected patient population, it was possible to analyze data by line of treatment. What became clear from this was that bevacizumab combinations were more effective when initiated earlier; overall RR was $62 \%$ in patients receiving bevacizumab as part of first-line therapy, $44 \%$ in those receiving second-line therapy, and $14 \%$ in those receiving the drug as part of later-line therapy; disease control rates by line of treatment were $95 \%, 93 \%$, and $76 \%$, respectively. Analysis of PFS by line of treatment found that the PFS in those patients receiving bevacizumab as part of first-line therapy was 11.7 months, which is similar to the 9.9 months and 10.8 months PFS reported, respectively, in the analyses of the BRITE (US) and FirstBEAT (Europe) observational registries. ${ }^{6,7}$ Efficacy of bevacizumab-containing therapy as second-line treatment reported here was at least comparable with published data of its use in combination with FOLFOX in second-line $\mathrm{mCRC}$, where PFS in patients who had already received an irinotecan-based regimen was only 7.9 months (versus 8.7 months in the present study), and OS was 12.3 months (versus 18.7 months in the present study). ${ }^{2}$ OS from initiation of treatment to death was 33 months for the selected patients receiving bevacizumab beyond progression, which is in line with the findings from the BRITE registry ( 32 months $)^{19}$ but longer than in the TML study (24 months). ${ }^{4}$

It would also appear from this retrospective observational study that use of bevacizumab in the initially unresectable appears to be feasible with promising early data. Initially, disease only patients with nonresectable disease and high tumor burden initiated bevacizumab-containing therapy. However, following bevacizumab plus chemotherapy, $9 \%$ of patients with initially unresectable disease became resectable, and $\mathrm{R} 0$ resection was achieved in $90 \%$. Due to concerns over possible bevacizumab-related complications during resection, bevacizumab treatment was interrupted 6-8 weeks before surgery and was restarted 4 weeks after resection. Morbidity was low with no bleeding or wound healing complications being observed with bevacizumab. Analysis of liver function showed that, 3 months after resection, livers had regenerated as anticipated with enzyme levels and synthesizing capacity returned to the baseline levels.

One major problem at liver resection is how to find microscopic residual metastases that are no longer detectable radiologically (radiologic complete response). In our material, $47 \%$ (31/66) of the original metastases had disappeared at preoperative radiologic evaluation. Peroperatively, $48 \%(32 / 66)$ of the original lesions could be found using ultrasound. In careful histological examination pathologic complete response was observed in $23 \%$ (7/31) of the removed lesions. The radiologic complete response lesions that could not be identified peroperatively usually recurred within 1 year, leading to re-resections in follow-up. Strikingly, the same patient may have totally necrotic lesions with $0 \%$ viable cells and with $90 \%$ viable tumor cells.

In conclusion, these efficacy and tolerability findings relating to bevacizumab-containing combination therapy administered to an unselected patient population are in line with the results of randomized studies in mCRC. Treatmentrelated toxicity was acceptable. Furthermore, liver resections may be performed safely among patients treated with bevacizumab.

\section{Disclosure}

Pia Osterlund has received honoraria for advisory boards, lecturing or consulting fees, and travel grants from Amgen, Bayer, Celgene, Lilly, Merck, Roche, and Sanofi, and research funding from Amgen, Roche, and Sanofi. Tuomo Alanko has received honoraria for advisory board membership, lecturing, consulting, and travel grants from Amgen, AstraZeneca, Bayer, Bristol-Myers Squibb, Celgene, Lilly, 
Pfizer, Pierre Fabre, Roche, and Sanofi. Petri Bono has had a role in Advisory Boards or given an expert testimony for GlaxoSmithKline, Pfizer, and Novartis. Helena Isoniemi has received honoraria for advisory board membership, lecturing and travel grants from Roche. Editorial assistance was provided by Miller Medical Communications Ltd, funded by Roche, Finland. Reetta Peltonen reports no conflicts of interest in this work.

\section{References}

1. Hurwitz H, Fehrenbacher L, Novotny W, et al. Bevacizumab plus irinotecan, fluorouracil, and leucovorin for metastatic colorectal cancer. N Engl J Med. 2004;350(23):2335-2342.

2. Giantonio BJ, Catalano PJ, Meropol NJ, et al. High-dose bevacizumab improves survival when combined with FOLFOX4 in previously treated advanced colorectal cancer: Results from the Eastern Cooperative Oncology Group (ECOG) study E3200. J Clin Oncol. 2007;25(12): 1539-144.

3. Kabbinavar FF, Hambleton J, Mass RD, Hurwitz HI, Bergsland E, Sarkar S. Combined analysis of efficacy: the addition of bevacizumab to fluorouracil/leucovorin improves survival for patients with metastatic colorectal cancer. J Clin Oncol. 2005;23(16):3706-3712.

4. Bennouna J, Sastre J, Arnold D, et al. Continuation of bevacizumab after first progression on metastatic colorectal cancer (ML18147): a randomized phase 3 trial. Lancet Oncol. 2013;14(1):29-37.

5. Saltz LB, Clarke S, Diaz-Rubio E, et al. Bevacizumab in combination with oxaliplatin-based chemotherapy as first-line therapy in metastatic colorectal cancer: A randomized phase III study. J Clin Oncol. 2008; 26(12):2013-2019.

6. Kozloff M, Yood MU, Berlin J, et al. Clinical outcomes associated with bevacizumab-containing treatment of metastatic colorectal cancer: the BRiTE observational cohort study. Oncologist. 2009;14(9):862-870.

7. Van Cutsem E, Rivera F, Berry S, et al. Safety and efficacy of first-line bevacizumab with FOLFOX, XELOX, FOLFIRI and fluoropyrimidines in metastatic colorectal cancer: the BEAT study. Ann Oncol. 2009;20(11): $1842-1847$.

8. Bendell JC, Bekaii-Saab TS, Cohn AL, et al. Treatment patterns and clinical outcomes in patients with metastatic colorectal cancer initially treated with FOLFOX-bevacizumab or FOLFIRI-bevacizumab: results from ARIES, a bevacizumab observational cohort study. Oncologist. 2012;17(12):1486-1495.
9. Skillings JR, Johnson H, Miller K, et al. Arterial thromboembolic events (ATEs) in a pooled analysis of 5 randomized, controlled trials (RCTs) of bevacizumab (BV) with chemotherapy. J Clin Oncol. 2005;23(Suppl):196s(Abst \#3019).

10. Hambleton J, Novotny WF, Hurwitz H, et al. Bevacizumab does not increase bleeding in patients with metastatic colorectal cancer receiving concurrent anticoagulation. J Clin Oncol. 2004;22(Suppl): 14S(Abst \#3528).

11. Lordick F, Geinitz H, Theisen J, Sendler A, Sarbia M. Increased risk of ischemic bowel complications during treatment with bevacizumab after pelvic irradiation: report of three cases. Int J Radiat Oncol Biol Phys. 2006;64(5):1295-1298.

12. Kozloff M, Hainsworth J, Badarinath S, et al. Management of hypertension (HTN) in patients with metastatic colorectal cancer treated with bevacizumab (BV) plus chemotherapy. Eur J Cancer. 2007;5(Suppl 4): 251(Abst \#3049).

13. Scappaticci FA, Fehrenbacher L, Cartwright T, et al. Surgical wound healing complications in metastatic colorectal cancer patients treated with bevacizumab. J Surg Oncol. 2005;91(3):173-180.

14. Gruenberger B, Scheithauer W, Tamandl D, Zielinski C, Schueller J, Gruenberger T. Effectiveness of neoadjuvant chemotherapy including bevacizumab in patients with resectable colorectal cancer liver metastases. J Clin Oncol. 2007;25(18S):4060.

15. Gruenberger T, Tamandl D, Schueller J, et al. Bevacizumab, capecitabine, and oxaliplatin as neoadjuvant therapy for patients with potentially curable metastatic colorectal cancer. J Clin Oncol. 2008;26(11):1830-1835.

16. D'Angelica M, Kornprat P, Gonen M, et al. Lack of evidence for increased operative morbidity after hepatectomy with perioperative use of bevacizumab: a matched case-control study. Ann Surg Oncol. 2007;14(2):759-765.

17. Bendell JC, Fernando M, Morse M, et al. A phase II study of oxaliplatin (OX), capecitabine (CAP), and bevacizumab (BV) in the treatment of metastatic colorectal cancer. J Clin Oncol. 2006;24(Suppl): 18S(Abst \#3541).

18. Sobrero A, Ackland S, Clarke S, et al. Phase IV study of bevacizumab in combination with infusional fluorouracil, leucovorin and irinotecan (FOLFIRI) in first-line metastatic colorectal cancer. Oncology. 2009;77(2):113-119.

19. Grothey A, Sugrue MM, Purdie DM, et al. Bevacizumab beyond first progression is associated with prolonged overall survival in metastatic colorectal cancer: results from a large observational cohort study (BRiTE). J Clin Oncol. 2008;26(33):5326-5334.
OncoTargets and Therapy

\section{Publish your work in this journal}

OncoTargets and Therapy is an international, peer-reviewed, open access journal focusing on the pathological basis of all cancers, potential targets for therapy and treatment protocols employed to improve the management of cancer patients. The journal also focuses on the impact of management programs and new therapeutic agents and protocols on

\section{Dovepress}

patient perspectives such as quality of life, adherence and satisfaction. The manuscript management system is completely online and includes a very quick and fair peer-review system, which is all easy to use. Visit http://www.dovepress.com/testimonials.php to read real quotes from published authors. 